\title{
Sustainable Curriculum to Increase Scholarly Activity in a Family Medicine Residency
}

Sajeewane Manjula Seales, MD, MPH; Robert P. Lennon, MD, JD; Kristian Sanchack, MD, MHA; Dustin K. Smith, DO

BACKGROUND AND OBJECTIVES: Scholarly activity (SA) is an Accreditation Council for Graduate Medical Education (ACGME) requirement for family medicine residency programs. Engaging residents in scholarly activity can be challenging. In 2010, the Naval Hospital Jacksonville Family Medicine Residency (NHJ FMR) program pioneered a research curriculum that dramatically increased resident SA output. The purpose of this study was to determine whether this output sustained over time.

METHODS: A retrospective records review was performed on resident SA at the NHJ FMR program between academic years 2012-2013 to 2016-2017 $(\mathrm{N}=185)$. The following research curriculum interventions were implemented over academic years 2010-2012: a faculty research coordinator position, a scholarly activity point system, and a peer-driven resident research coordinator position. SA output was calculated based on total resident projects per year and "quality projects" or peer-reviewed projects per year. Regression analysis and Mann-Whitney $U$ test tested nonparametric group comparisons.

RESULTS: The number of quality projects per resident per year increased from 0.34 in 2012-2013 to 1.05 in the 2016-2017 academic year. The quality projects per resident per year demonstrated a statistically significant increase over time $(F(1,9)-18.98, P<.005, \mathrm{R} 2$ of 0.6784$)$. When comparing preintervention years to postintervention years the average quality projects per resident was statistically significant $(P<.005)$.

CONCLUSIONS: This curriculum model emphasizes unique and reliably sustainable interventions to increase scholarly output that can be implemented at any residency program. SA volume and quality increased over 5 postintervention years despite annual resident research coordinator turnover. This research demonstrates a resident-driven culture change that warrants future research on adaptability to other programs.

(Fam Med. 2019;51(3):271-5.)

doi: 10.22454/FamMed.2019.906164
$\mathbf{R}$ esident scholarly activity (SA) promotes better patient care, resident satisfaction, and future careers in academic medicine. ${ }^{1-8}$ After the Accreditation Council for Graduate Medical Education (ACGME) implemented new requirements for resident SA, family medicine residency programs developed various strategies to increase SA. ${ }^{3}$ Implementation of a research track, faculty research coordinator (FRC), research curriculum, research day, protected research time, and recognition for scholarship are all strategies that increase resident SA. ${ }^{4}$

New research supports a residentdriven effort in the form of a "resident research coordinator." ${ }^{1,5} \mathrm{We}$ previously reported a dramatic increase in resident SA attributable to a resident research coordinator. ${ }^{1}$ Feedback following this initial study questioned the sustainability of this improvement given annual resident research coordinator turnover; no study to date examines whether this intervention has a lasting impact. We evaluated the sustainability of resident research coordinator-driven improvement in resident SA over a 5 -year period.

From Naval Hospital Jacksonville, Jasksonville, FL (Drs Seales, Lennon, and Sanchack), Naval Branch Health Clinic Diego Garcia (Dr Smith). 


\section{Methods}

Starting in 2010, we implemented three interventions at the Naval Hospital Jacksonville (NHJ) Family Residency Program over 2 academic years. This military program is accredited to 39 residents with off-cycle residents bringing totals as high as 45 residents per year. Prior to the interventions, residents completed SA to graduate, but it was loosely defined, ranging from internal process improvement to Institutional Review Board (IRB)-approved studies.

We implemented an FRC position and SA point system during academic year 2010-2011. Ideally, the FRC would have a wealth of research experience and leadership skills. Although our FRC has limited research experience and holds the academic rank of assistant professor, they were able to function effectively with a 0.1 FTE deduction per week dedicated to the position. The FRC identifies venues for research, liaises between residents and faculty, and maintains the SA point system adapted from Seehusen et al (Table 1). ${ }^{9}$ The point system establishes a benchmark for what SA meets graduation requirements and incentivizes residents creating competition. The resident with the greatest accumulated points receives an award at graduation.

We implemented a resident research coordinator position in 20112012 as a motivating, mentoring, and research force-multiplier. The position requires a PGY-2 or PGY-3 resident with strong research interest and drive to further peer scholarship. Interested residents submit a letter of intent to the program director and chief residents who select the most qualified out of one to three applicants per year. Since the position adds to preexisting resident responsibilities, it tends to self-identify in that only residents amenable to performing this extra work volunteer. The resident research coordinator helps residents identify scholarly projects, teaches a case report workshop, and guides residents through the process of IRB approval, conference application, and publication submission. The resident research coordinator intermittently receives a dedicated half day of research, although much of the work is done through asynchronous communication, informal discussions, or during one half day of didactics per week blocked off for all residents. This flexibility allows for minimal interruption in their schedule and all residents who served in this role easily met the ACGME requirements for graduation. Each resident research coordinator shadows their predecessor and receives on-the-job training before assuming the position. At the completion of their tenure, this resident receives an award for their efforts. We did not survey time spent by these residents on their duties.

This is a retrospective review of all resident SA from academic years 2012-2013 through 2016-2017 $(\mathrm{N}=185$ residents), 5 years after implementing the first resident research coordinator. Four different

Table 1: Research Point System*

\begin{tabular}{|c|c|}
\hline Scholarly Activity & Value \\
\hline Completion of an IRB-approved research project or a publishable well-conducted process improvement project & 10 \\
\hline $\begin{array}{l}\text { Acceptance of a manuscript describing a case report, clinical review, or research project in a peer-reviewed } \\
\text { medical journal }\end{array}$ & 8 \\
\hline Acceptance for publication of an Family Physicians Inquiries Network (FPIN) Clinical Inquiry & 7 \\
\hline Acceptance for publication of a 5-minute Clinical Consult & 7 \\
\hline Podium presentation at a regional, national, or international medical conference & 7 \\
\hline $\begin{array}{l}\text { Poster presentation at a regional, national or international medical conference for a case report or original } \\
\text { research }\end{array}$ & 6 \\
\hline Acceptance for publication of a FPIN Help Desk Answer or Electronic Medical Reference (eMedRef) & 5 \\
\hline $\begin{array}{l}\text { Submission without acceptance of a manuscript describing a case report, clinical review or research project in a } \\
\text { peer-reviewed medical journal }\end{array}$ & 5 \\
\hline Acceptance for publication of a Letter to the Editor in a peer-reviewed journal & 3 \\
\hline Being recognized at a local, regional, national or international conference & 3 \\
\hline Publications for lay public such as newspaper or magazine articles on medical topics & 2 \\
\hline Presentation at the Southeast Regional Annual Resident Research Symposium & 2 \\
\hline Presentation at Grand Rounds/Tracer Interdisciplinary Morbidity and Mortality conference to hospital staff & 1 \\
\hline Submission without acceptance of a presentation at a regional, national, or international conference & 1 \\
\hline Presentation of case at Tumor Board & 1 \\
\hline Completion of CITI/IRB training & 1 \\
\hline Presenting at Journal Club (voluntary or mandatory) & 1 \\
\hline
\end{tabular}

* Adapted from Seehusen et al. ${ }^{9}$ 
residents held the position during this time period. We tracked total SA and "quality" projects. In the previous review, quality projects were defined as projects presented at a regional or higher-level conference or published in a peer-reviewed journal. ${ }^{1}$ In this study, we adopt a more stringent definition of quality projects as projects presented at a regional or higher-level conference or published in a PubMed-indexed, peer-reviewed journal with a Medline number. This change ensures quality projects undergo more rigorous, standardized peer review. This project received IRB approval.

\section{Results}

Using the less stringent definition, in the 3 academic years prior to the interventions, six quality projects were produced. The average number of quality projects per resident per year was 0.07 . The first academic year the resident research coordinator position was implemented, the number of quality projects per resident per year increased 13-fold to 0.91 . SA remained robust from that point forward (Figure 1). Total SA projects per resident per year increased from 1 to 2.61 over the 5 academic years. Using the more stringent interpretation, quality projects per resident increased from 0.34 in 2012-2013 to 1.05 in 20162017 (Figure 2). A linear regression of all years from preintervention to 5 years postintervention revealed a statistically significant positive slope of quality projects per resident $\left(F(1,9)-18.98, P<.005, R^{2}\right.$ of 0.6784 ; Figure 3). A Mann-Whitney $U$ test of preintervention years to postintervention years was also significant $\left(U=0, \mathrm{n}_{1}=5, \mathrm{n}_{2}=6, z=2.64733 P<.05\right)$.

\section{Discussion}

We saw a sustained increase in resident SA output after the research curriculum interventions. Even with the stricter definition, every postintervention year is higher than any preintervention year SA.
One hundred percent of our residents meet ACGME SA graduation requirements. Currently, residents produce just over one quality project every year, exceeding ACGME requirements of $1 \mathrm{SA}$ project over 3 years and faculty requirements of 2 research projects over 5 years. ${ }^{3}$ Our previous report demonstrated correlation between the resident research coordinator and increased SA. This study shows that the increase was sustained, suggesting that this peerdriven mentorship bred a change in residency culture. SA is no longer a box to check for graduation, but an active part of each resident's entire educational experience.

Any form of leadership can improve resident SA. ${ }^{4,10}$ Barriers to implementing a FRC include limited faculty research expertise at a small program, funds to sustain a position, and the approachability of said faculty. ${ }^{6}$ Ledford et al also describe uncertainty barriers that contribute to learner anxiety in resident scholarship including uncertainty

Figure 1: Quality Projects Before and After Research Curriculum Interventions

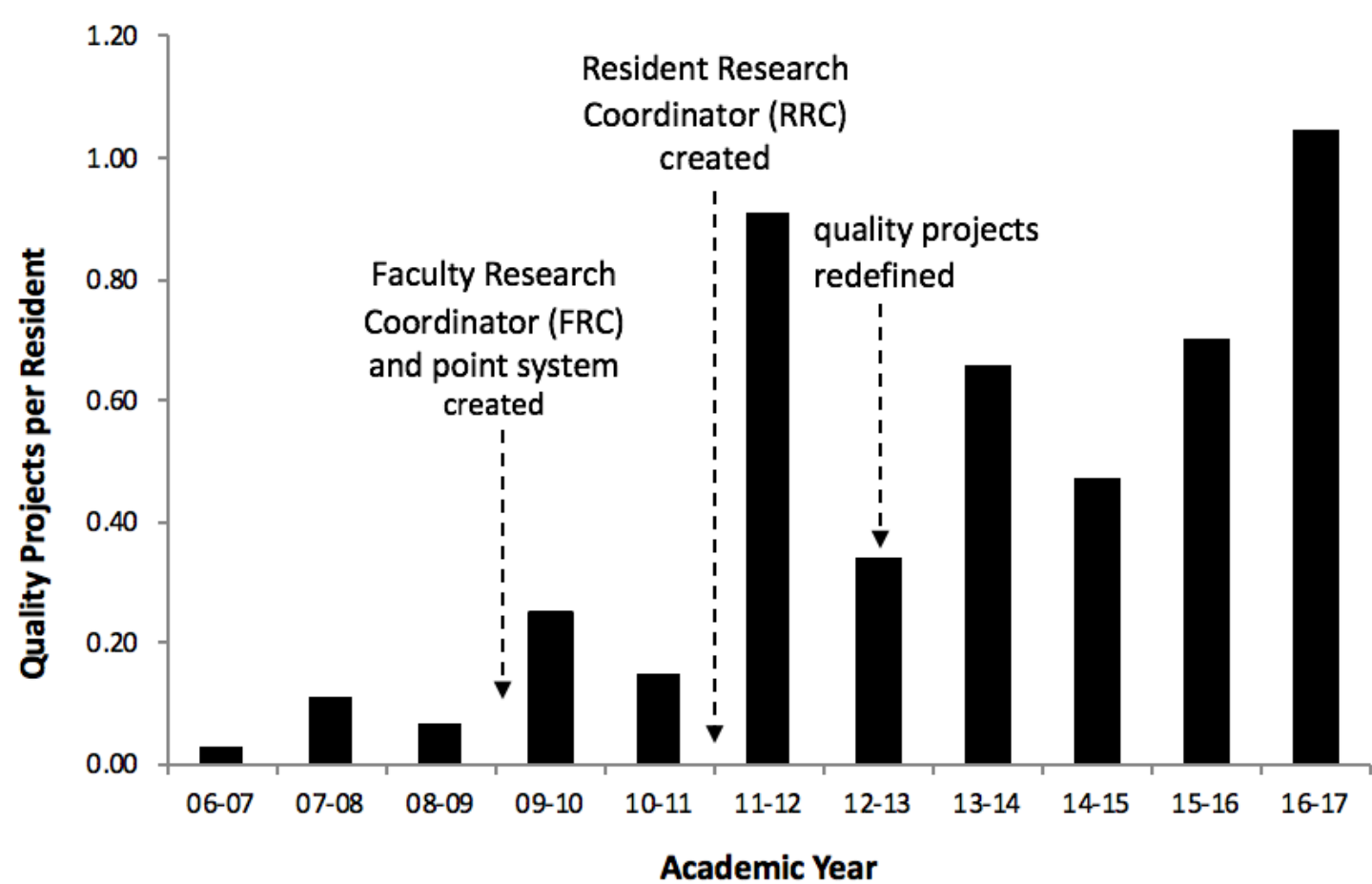

Note: Academic years 2012-2013 to 2016-2017 use a stricter "quality project" definition than years 2006-2011. 
Figure 2: Scholarly Activity (SA) per Resident per Academic Year

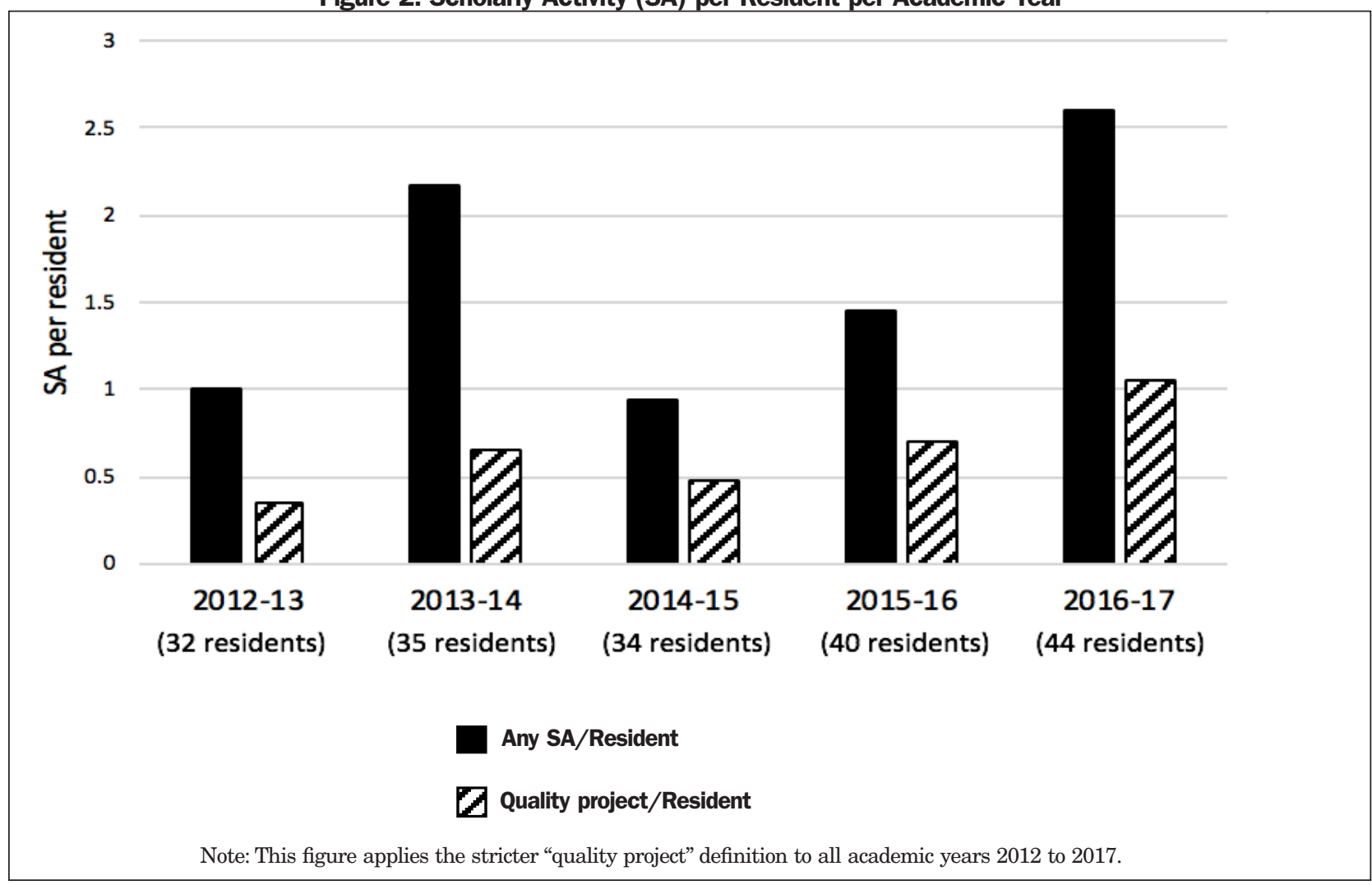

Figure 3: Plot of Quality Projects per Resident

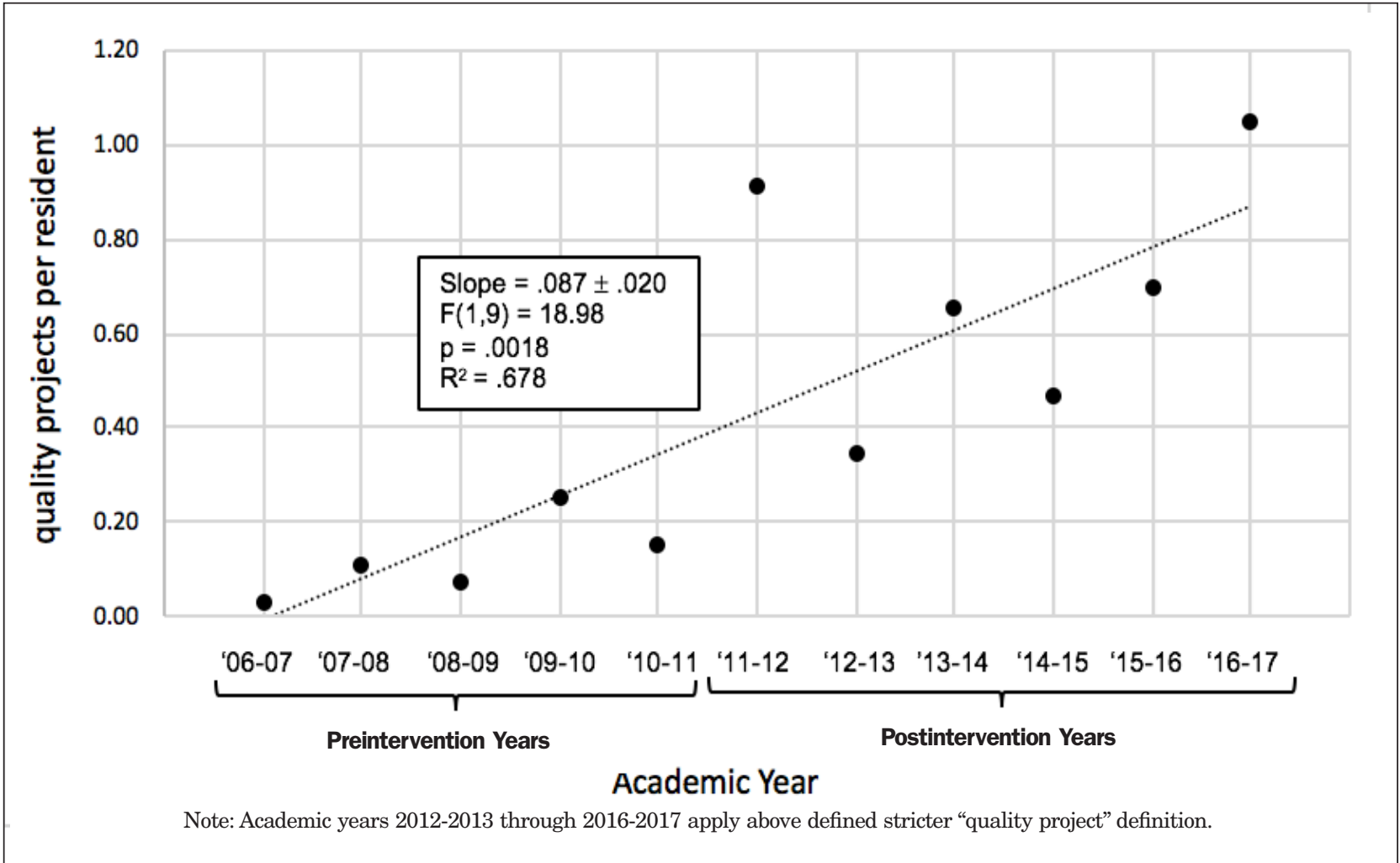


about their identity as a clinician, the protocols and norms of scholarship, and the establishment of a mentor relationship. ${ }^{11}$ The resident research coordinator addresses all of these barriers. As a stakeholder in resident research they are both participant and guide. While research experience is valued, no formal research training is required and motivation and perseverance continue to be the primary traits we use to identify each candidate. Hoedebecke et al also confirmed in a 1-year study of a resident research coordinator that residents report increased resident interest and enthusiasm from direct and visible peer leadership. ${ }^{5}$ Finally, no funds are required and it does not detract from a faculty position.

This study is limited by its design as a records-review at a single program. Although this study was performed at a military residency, the results are applicable to nonmilitary programs. None of the interventions are military-specific and the program operates under the same ACGME and American Board of Family Medicine requirements. FTE deductions would have to be strictly defined for faculty, and military awards would have to be adjusted to a civilian setting. Also, we cannot accurately determine the relative impact of each intervention as there was no significant interval between the FRC, point system, and resident research coordinator position..$^{12}$ Qualitatively, we believe that the resident research coordinator had the most significant and long-lasting impact because the most explosive growth in SA occurred the year the resident research coordinator started with 5 subsequent years of sustained increase in total and quality SA projects despite annual turnover of the position.

No one research curriculum intervention is more effective than others and individual programs should perform a needs assessment to identify barriers to SA output. ${ }^{12}$ In addition to the volume of production described, it has been our experience that the resident research coordinator increased enthusiasm and interest in other residents to complete SA via a grassroots approach. This unique peer-driven intervention demonstrates a dramatic and sustainable increase in resident SA that is adaptable to any residency program.

ACKNOWLEDGMENTS: This study was presented and awarded first place for Educational Research at the Uniformed Services Academy of Family Physicians Annual Meeting, March 17, 2018 in Jacksonville, Florida.

DISCLAIMER: The opinions and assertions contained herein are the private views of the authors and are not to be construed as official or as reflecting the views of the Uniformed Services University of the Health Sciences, the US Navy, the US Air Force or the US Department of Defense at large.

CORRESPONDING AUTHOR: Address correspondence to Kristian Sanchack, MD, MHA, Naval Hospital Jacksonville, 2080 Child Street, Jacksonville, FL 32214. 904-616-5591. Fax: 904-542-4381. kristian.e.sanchack.mil@mail.mil.

\section{References}

1. Lennon RP, Oberhofer AL, McNair V, Keck JW. Curriculum changes to increase research in a family medicine residency program. Fam Med. 2014;46(4):294-298

2. Simasek M, Ballard S, Phelps P, et al. Meeting resident scholarly activity requirements through a longitudinal quality improvement curriculum. J Grad Med Educ. 2015;7(1):86-90.

3. Accreditation Council for Graduate Medical Education. Scholarly Activity Guidelines Review Committee for Family Medicine. 2016. https://www.acgme.org/Portals/0/PFAssets/ ProgramResources/120_scholarly-activityguidelines.pdf. Accessed December 1, 2017.

4. Stevenson MD, Smigielski EM, Naifeh MM Abramson EL, Todd C, Li ST. Increasing scholarly activity productivity during residency: a systematic review. Acad Med. 2017;92(2):250266.

5. Hoedebecke K, Rerucha C, Runser L. Increase in residency scholarly activity as a result of resident-led initiative. Fam Med. 2014;46(4):288-290.

6. Marshall KE, Hammill TL. Research 101: An Initiative to Encourage and Facilitate Quality Resident Research in a Military Setting. Otolaryngol Head Neck Surg. 2017;156(6):10541059

7. Pound CM, Moreau KA, Ward N, Eady K, Writer H. Enhancing pediatric residents' scholar role: the development of a Scholarly Activity Guidance and Evaluation program. Med Educ Online. 2015;20(1):27452-27456.

8. Hayward RA, Taweel F. Data and the internal medicine houseofficer: alumni's views of the educational value of a residency program's research requirement. J Gen Intern Med. 1993;8(3):140-142.
9. Seehusen DA, Asplund CA, Friedman M. A point system for resident scholarly activity. Fam Med. 2009;41(7):467-469.

10. Chen JX, Kozin ED, Sethi RKV, Remenschneider AK, Emerick KS, Gray ST. Increased Resident Research over an 18-Year Period: A Single Institution's Experience. Otolaryngol Head Neck Surg. 2015;153(3):350-356.

11. Ledford CJW, Seehusen DA, Villagran MM, Cafferty LA, Childress MA. Resident scholarship expectations and experiences: sources of uncertainty as barriers to success. J Grad Med Educ. 2013;5(4):564-569.

12. Alweis R, Wenderoth S, Donato A. Effectiveness of iterative interventions to increase research productivity in one residency program. J Community Hosp Intern Med Perspect. 2015;5(6):29203.

13. Robbins MS, Haut SR, Lipton RB, et al. A dedicated scholarly research program in an adult and pediatric neurology residency program. Neurology. 2017;88(14):1366-1370.

14. Ahmad F, Ferrari M, Moravac C, Lofters A, Dunn S. Expanding the meaning of being a peer leader': qualitative findings from a $\mathrm{Ca}-$ nadian community-based cervical and breast cancer screening programme. Health Soc Care Community. 2017;25(2):630-640.

15. Andre C, Deerin J, Leykum L. Students helping students: vertical peer mentoring to enhance the medical school experience. BMC Res Notes. 2017;10(1):176. 